\title{
Evolution of the edible oil supply chain in Cuba
}

\section{Yinef Pardillo Baez ${ }^{1}$, Dianelys Milagros Ruíz Alvarez ${ }^{2}$, Abel González-Carvajal Alberto ${ }^{3}$, Martha Inés Gómez Acosta ${ }^{4}$}

1,2,3,4 Instituto Superior Politécnico José Antonio Echeverría (CUJAE). Ciudad de la Habana, Cuba.

Email: yinef@ind.cujae.edu.cu,dianelysra@ind.cujae.edu.cu,abel@ind.cujae.edu.cu,marthagom@tesla.cujae.edu.cu

Received: December $11^{\text {th }}, 2017$.

Accepted: February $01^{\text {th }}, 2018$.

Published: March $30^{\text {th }}, 2018$.

Copyright (C2016 by authors and Institute of Technology Galileo of Amazon (ITEGAM).

This work is licensed under the Creative Commons Attribution International

License (CC BY 4.0).

http://creativecommons.org/licenses/by/4.0/ (c) (1) (3)

\section{Opea Actese}

\begin{abstract}
The edible oil is considered one of the stars products in the cuban national market, as a result of its discharge it demands for the population and for the industries that use it as matter it prevails in its productive process [1]. Since the year 2005 the Supply Chain of Edible Oil has come carrying out investigations with the objective of elevating the levels of service to its final client. Following this purpose is made in this year an association between the Laboratory of Logistics and Management of the Production (LOGESPRO) and the Enterprise of the Oils ECASOL, coordinating entity of the Chain. In the beginnings of the work with ECASOL the emphasis is made in the availability studies of edible oil, and after to the application of the Value Network Model, later is making wider the field of action of the investigations to develop between LOGESPRO and ECASOL. The objective of the present case consists on analyzing the evolutionary development that has suffered the Supply Chain of Edible Oil in CUC with ECASOL like coordinating entity of the same one starting from the achieved association.
\end{abstract}

Keywords: Supply Chains, Edible oil, Value Network Model.

\section{Evolución de la cadena de suministro de aceite comestible en Cuba}

\section{RESUMEN}

El aceite comestible es considerado uno de los productos estrellas en el mercado nacional cubano, debido a su alta demanda en la población y las industrias que lo emplean como materia prima en su proceso productivo [1]. En la Cadena de Suministro de Aceite Comestible se vienen realizando investigaciones desde el año 2005 con el objetivo de elevar los niveles de servicio a su cliente final. Siguiendo este propósito se crea en el propio año una alianza entre el Laboratorio de Logística y Gestión de la Producción (LOGESPRO) y la Empresa Comercializadora de Aceites ECASOL, entidad coordinadora de la Cadena. En los inicios del trabajo con ECASOL se hace énfasis en los estudios de disponibilidad de aceite comestible, pasando posteriormente a la aplicación del Modelo de Redes de Valor y así sucesivamente a un campo de acción cada vez más amplio en el marco de las investigaciones entre LOGESPRO y ECASOL. El objetivo del presente caso consiste en analizar el desarrollo evolutivo que ha sufrido la Cadena de Suministro de Aceite Comestible Comercializado en CUC con ECASOL como ente coordinador de la misma a partir de la alianza lograda.

PALABRAS CLAVES: Cadena de Suministro, Aceite Comestible, Modelo de Red de Valor.

\section{INTRODUCCIÓN}

La Gestión Integrada de las Cadenas de Suministro (GICS) está tomando cada vez mayor importancia debido al desarrollo comercial, logístico y tecnológico alcanzado en la sociedad actual y la globalización existente en el mundo, debido a que hoy no se concibe la competencia entre empresas aisladas, pues según plantean Mangan, Aguilar y Anaya hoy no son las empresas y productos los que compiten de forma individual, sino las cadenas de suministro coordinadas [2-4].

Hoy la logística y la gestión de la cadena de suministro constituyen tendencias prioritarias en la formulación de las 
estrategias de las empresas, y como plantea Pardillo se impone la necesidad de crear una cultura de gestión interorganizacional. Según Flynn, muchos investigadores han tratado ampliamente la necesidad de una relación integrada entre productores y socios en la cadena de suministro, pero solo recientemente ha habido un enfoque sistemático de integración de la cadena de suministro [5].

Muchos autores han conceptualizado el término cadena de suministro, Gómez y Acevedo, Ivanov y Vitasek coinciden en que es una red global usada para suministrar productos y servicios desde la materia prima hasta elb cliente final a través de un flujo diseñado de información, distribución física, y efectivo [6-8]. Según Harrison, una cadena de suministro es la red de socios quienes colectivamente convierten un producto básico en un producto final que es valorado por el cliente final, y es quien maneja los retornos a cada actor [9]. Por su parte la Red de Valor constituye una etapa superior de desarrollo en las cadenas de suministro y Acevedo al definirla hace mención a una red integrada de procesos de agregación de valor desde el proveedor primario hasta el consumidor [10].

El propio desarrollo de la investigación permite a la autora Pardillo replantear el concepto de cadena de suministro, siendo su definición la base de este trabajo. El concepto abordado plantea que una cadena de suministro es un nexo de procesos en los que participan un conjunto de entidades desde los proveedores primarios hasta el cliente final, donde se interrelacionan flujos de materiales, información y efectivo, con el objetivo de satisfacer las demandas de los clientes de manera eficiente, eficaz y competitiva, considerando la adecuada preservación y mejoramiento del medio ambiente [1].

En el marco de la actualización del Modelo Económico Cubano se establece en Cuba cada vez más, la necesidad del fortalecimiento de la integración de las cadenas productivas, con el objetivo de sustituir importaciones y lograr resultados económicos favorables, aprovechando para ello las capacidades productivas con que cuenta el país. Esta linea de acción queda recogida en el Lineamiento número 36 de la Política
Económica y Social del Partido y la Revolución el cual establece: "En la actividad agroindustrial, se impulsará en toda la cadena productiva la aplicación de una gestión integrada $(\cdots)$ orientada al incremento de la producción de alimentos $(\cdots)$, incluyendo el perfeccionamiento de los servicios a los productores, con reducción de costos, el mayor empleo de componentes e insumos de producción nacional y del aprovechamiento de las capacidades científico-tecnológicas disponibles en el país" [11].

Dada la condición actual de dualidad monetaria en Cuba, donde coexiste el CUP (peso cubano) con el CUC (moneda libremente convertible), la Cadena de Suministro de Aceite Comestible en el país se desarrolla a través de dos ramas fundamentales, cada una relacionada con los tipos de monedas planteadas. Las investigaciones que se han desarrollado por parte de LOGESPRO fundamentalmente han estado relacionadas con la cadena asociada al CUC y por tanto es en esta rama donde mayores resultados se han alcanzado.

A partir de la labor realizada por el equipo de trabajo LOGESPRO-ECASOL, la Cadena de Suministro de Aceite Comestible Comercializado en CUC, se ha convertido en referencia para otras cadenas en Cuba, debido al nivel de conocimiento, integración y comunicación que se ha ido alcanzando por parte de los actores de la cadena con su ente coordinador, la Empresa ECASOL. Con el despliegue de este caso se pretende precisamente, analizar el desarrollo evolutivo que se ha logrado en dicha cadena partiendo de la alianza creada entre LOGESPRO y ECASOL.

\section{DESARROLLO EVOLUTIVO DE LA CADENA D SUMINISTRO DE ACEITE COMESTIBLE}

La representación simplificada de la Cadena de Aceite Comestible Comercializado en CUC se puede observar en la figura 1 que se muestra seguidamente.

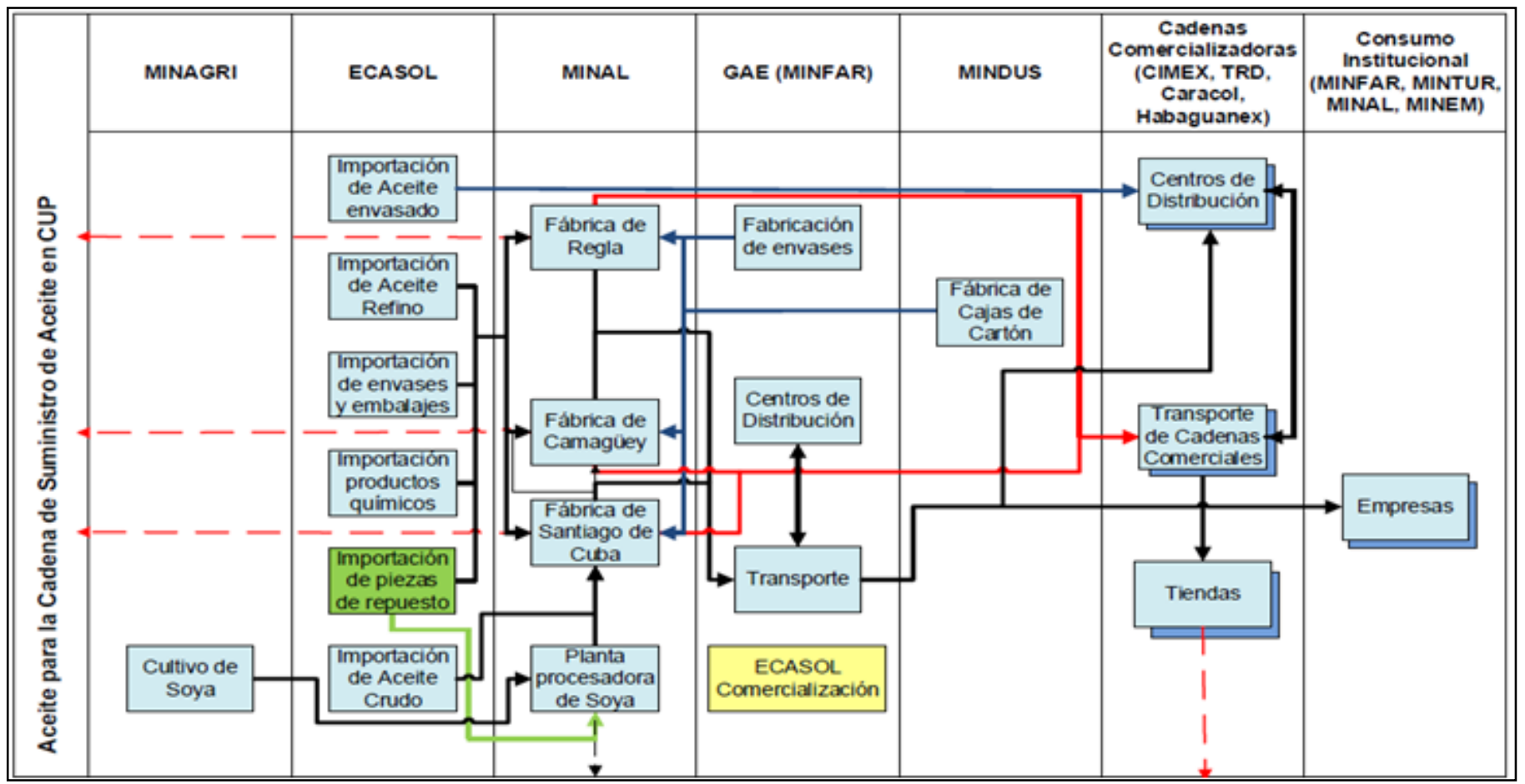

Figura1: Cadena de Suministro de Aceite Comestible Comercializado en CUC.

Fuente: Los autores, (2018). 


\section{II.1 ETAPAS INICIALES}

A partir del año 2005 ECASOL y LOGESPRO establecen estrechos lazos de colaboración en función de estudiar, desarrollar e implementar novedosos conceptos teórico-prácticos en el campo de la gestión empresarial, logística y de las cadenas de suministro en el país. En estos momentos, la comercialización de aceite comestible en CUC se encuentra muy deprimida, presentando como problemas fundamentales la baja disponibilidad de sus productos en los puntos de consumo y por consiguiente valores bajos de ventas.

Desde el comienzo de la colaboración LOGESPROECASOL el centro de atención de la gestión de la empresa ha estado enfocado en buscar la satisfacción de los clientes finales, más que la de los clientes inmediatos de ECASOL, con el objetivo de incrementar las ventas totales de aceite en la cadena de suministro. Para demostrar la validez de este supuesto las primeras acciones desplegadas en el marco de la colaboración se centran en determinar los niveles de disponibilidad del aceite en los puntos de venta de las diferentes cadenas comercializadoras.

Como resultado del estudio de disponibilidad que marca el punto de partida en los trabajos realizados entre las dos organizaciones, en el propio año 2006, se obtiene que el nivel de disponibilidad de productos en los puntos de ventas a la población era muy bajo, presentando un valor de $26.4 \%$ considerando al aceite como genérico.

La principal conclusión del estudio es la no existencia de coordinación entre todos los eslabones que conforman la cadena de suministro, dado que los niveles de disponibilidad hacia atrás en los eslabones de la cadena de suministro son significativamente superiores, tal y como se muestra en la figura 2.

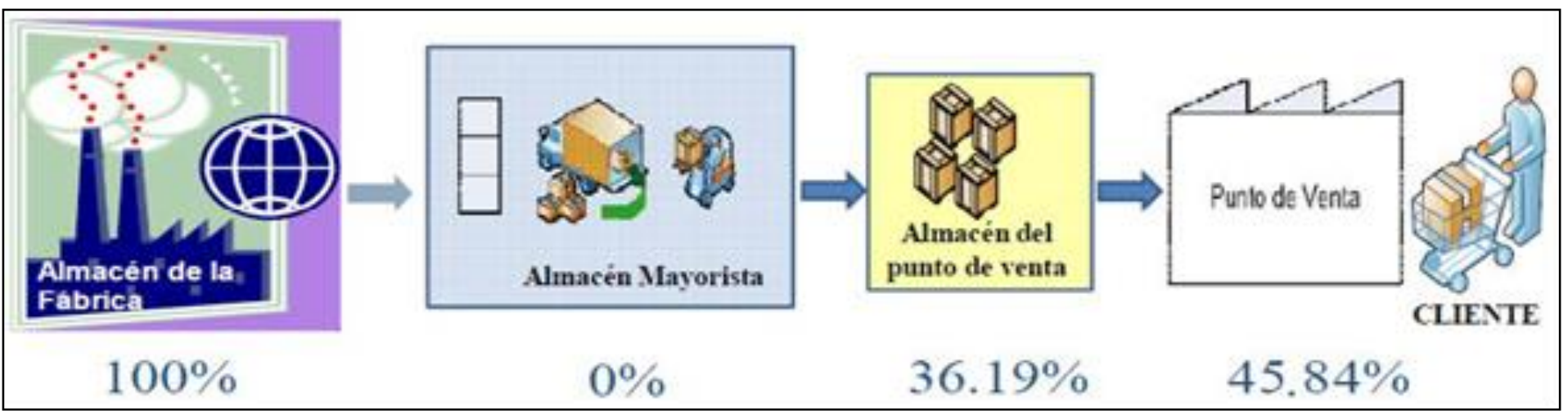

Figura 2: Porcentaje de disponibilidades de aceite en los actores de la Cadena de Suministro.

Fuente: [2].

Como se aprecia en la figura 1, en fábrica se presenta el $100 \%$ de disponibilidad de aceite comestible, sin entrar en el detalle de los diferentes surtidos, sin embargo, en los almacenes mayoristas, siguiendo el mismo criterio, es $0 \%$ la disponibilidad, mientras que en el punto final de la cadena de cara al cliente, lo que se percibe es solo un $45,84 \%$ de disponibilidad de los productos. Del gráfico anterior por tanto, se puede deducir que en buena medida los bajos niveles de disponibilidad en los puntos de venta se deben a una deficiente logística e inadecuada coordinación entre los actores de la cadena.

Es entonces que surge la necesidad de que un eslabón de la cadena tome el papel de coordinador de la misma para lograr la integración. Según plantea Pardillo, en una cadena de suministro productiva el papel coordinador debe caer en manos del eslabón productivo [13]. No obstante al no estar creadas las condiciones para ello, es decir al depender las industrias de Regla, Camagüey y Santiago de la gestión de ECASOL y al encontrarse las ventas de este último afectadas por los bajos niveles de disponibilidad, la dirección de la empresa toma la decisión de convertirla en la entidad focal o coordinadora de la cadena.

A partir de este momento en que ECASOL asume el papel de coordinador de la cadena de suministro de aceite comestible comercializado en CUC, la misma emprende en el propio año 2006 la aplicación de la Gestión Integrada de la Cadena de Suministro en base al Modelo de Red de Valor desarrollado por LOGESPRO. Para cumplir el papel de coordinador, la empresa despliega un conjunto de acciones de mejoramiento y cambios en su organización interna, en aras de lograr la integración con las otras entidades de la cadena de suministro.

Todas estas acciones se desarrollan bajo la dirección y seguimiento del director de ECASOL, el cual asume el liderazgo de la organización, cumpliendo de esta forma con uno de los siete pilares básicos de las organizaciones exitosas en Cuba [14].

Las acciones desarrolladas se enmarcan en tres etapas de desarrollo. Estas son:

\section{II.1.1 PRIMERA ETAPA}

Contenido: Centrada en la organización e integración interna de la empresa coordinadora para ponerla en condiciones de ejercer esa función de forma efectiva. Esta etapa busca el cumplimiento de los requerimientos básicos contenidos en el Decreto Ley 281 de 2007 referente al Perfeccionamiento Empresarial [15].

\section{Acciones:}

1. Completamiento y mejora del sistema de perfeccionamiento empresarial.

2. Implantación de la gestión por procesos.

3. Implantación y certificación del sistema de calidad de los procesos de realización del producto de la empresa.

4. Definición del papel y las funciones de ECASOL como centro de coordinación.

5. Informatización de la gestión económica y comercial de los procesos de compras y ventas. 


\section{II.1.2 SEGUNDA ETAPA}

Contenido: Centrada en la organización de la integración de la cadena de suministro.

Acciones:

1. Modificación del objeto social convirtiendo a la empresa ECASOL de una comercializadora de aceite vegetal comestible, en una empresa de manejo de productos desde la importación de materias primas y materiales, equipos, piezas de repuesto hasta el aceite envasado en almacenes de destino mayoristas de los clientes.

2. Ampliación de la licencia de importaciones para satisfacer el nuevo objeto social.

3. Calificación de los directivos de la empresa en los temas de manejo de cadena de suministros.

4. Definición y determinación de las relaciones de las entidades que intervienen en la cadena de suministro para el mercado de aceite comestible en CUC.

5. Diseño de las actividades y funciones de los procesos que intervienen en el manejo de cadena de suministro.

6. Modelo de organización de las actividades y funciones de la cadena de suministro.

\section{II.1.3 TERCERA ETAPA}

Contenido: Centrada en consolidar las bases y técnicas para realizar la gestión integrada en la cadena de suministro.

\section{Acciones:}

1. Gestión integrada de la cadena de suministro de ECASOL como centro coordinador con las entidades que intervienen en la cadena de suministro, a través de contratos de suministros, de servicios tercerizados y alianzas.

2. Crear la reserva para contingencia en almacenes subcontratados en calidad de depósito por ECASOL.

3. Introducción de las actividades de Inteligencia Comercial, administración de conocimientos, incluyendo la conexión de los sistemas informativos de los clientes.

4. Incorporar la gestión por valores.

5. Implantación y certificación del Sistema Integrado de Gestión de la Calidad, gestión ambiental y seguridad y salud del trabajo.

6. Elevación gradual de las competencias del personal involucrado en el Proyecto.

\section{II.2 PRINCIPALES RESULTADOS ALCANZADOS EN LAS ETAPAS INICIALES}

A partir de la implementación del Modelo de Red de Valor en la Cadena de Suministro de Aceite Comestible

Comercializado en CUC se comienzan a notar un conjunto de cambios. Entre los cambios fundamentales se aprecia el aumento sustancial de los niveles de disponibilidad de aceite en los puntos de venta y a su vez el incremento de los niveles de ventas de ECASOL a sus clientes inmediatos.

De un valor de disponibilidad de un $26,4 \%$ en el año 2006 , se puede constatar en la figura 3 como este indicador ha ido aumentando paulatinamente hasta presentar valores superiores a la media de disponibilidad en América Latina a partir del año 2011.

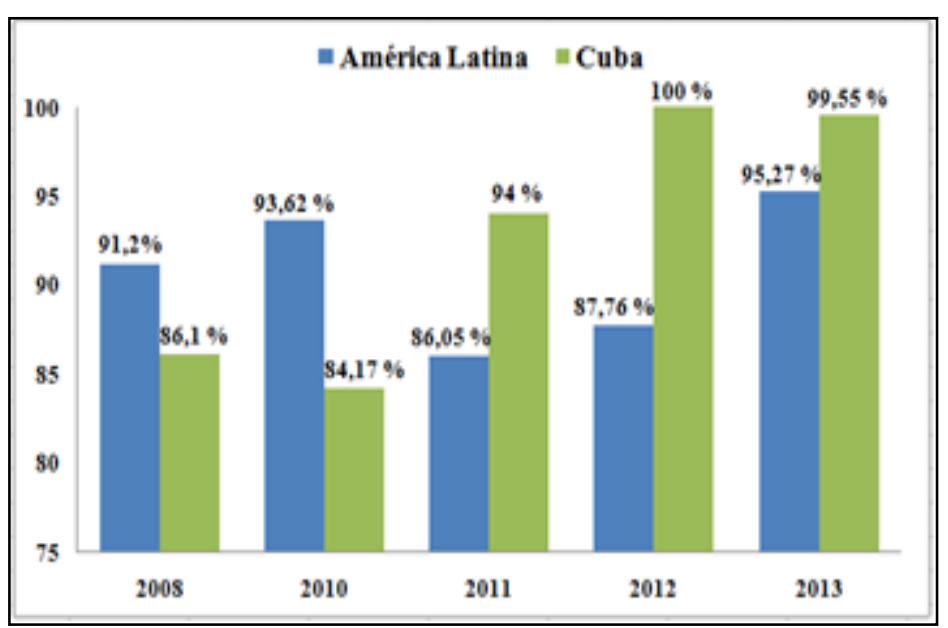

Figura 3: Disponibilidad de aceite por genérico en Cuba y América Latina.

Fuente: Los autores, (2018).

Junto al aumento de la disponibilidad de aceite en el punto de venta se produce además, un crecimiento de las ventas de ECASOL a sus clientes a partir del año 2008, año en el cual se logra un salto cuantitativo considerable en el indicador de disponibilidad. Esta situación se refleja en la figura 4.

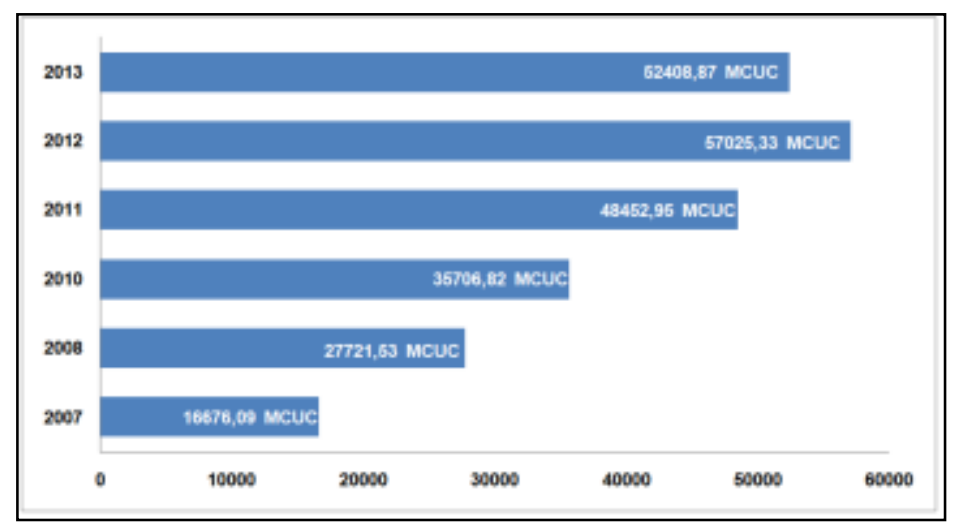

Figura 4: Importe total de Ventas en MCUC de ECASOL a sus clientes.

Fuente: Los autores, (2018).

Cuando se analiza la relación Disponibilidad-Ventas se puede apreciar que el incremento de la primera variable representa un incremento de la segunda. Esto ha sido comprobado a través de la dócima del coeficiente de correlación lineal de Pearson, el cual arroja un valor de 0,839. Este valor denota la existencia de una relación lineal fuerte y directa entre estas variables, es decir si aumenta la disponibilidad aumentan las ventas y si disminuye la primera, lo hace también la segunda variable.

En la Cadena de Aceite hasta el momento se ha implementado el Modelo de Red de Valor en un 50\%, lo que ha representado para ECASOL un aumento de las ventas en 31402 MCUC.

Los principales resultados alcanzados en cada una de las etapas, pueden resumirse de la siguiente forma:

\section{II.2.1 PRIMERA ETAPA (2005-2008)}

- $\quad$ Fijación del indicador de disponibilidad de productos a laventa como un medidor básico de la gestión de la cadena de suministro. 
- Primera representación de la cadena de suministro de aceite vegetal comestible en el país, la que constaba de 7 niveles fundamentales: proveedores, fábricas de refinación, almacén de productos terminados, almacenes nacionales, almacenes provinciales, almacenes municipales y clientes. Se advierte la importancia y necesidad de lograr un acercamiento con las fábricas refinadoras como eslabón clave de la cadena.

- Decisión de que ECASOL asuma el papel de coordinador de la rama de comercialización en CUC.

- Primer análisis del nivel de integración de la cadena, a partir del cual se comprueban los bajos niveles de estabilidades de los procesos a lo largo de la cadena.

- Primeras aproximaciones a las cadenas comerciales donde se va introduciendo la necesidad de aumentar la disponibilidad del producto y establecer políticas de surtidos para los puntos de venta. De este acercamiento también se desprenden los primeros análisis de factibilidad de distribución del aceite, para la cadena comercial CIMEX, comparando las alternativas de distribución a puntos de venta directamente desde fábrica o a través del centro de distribución de la cadena comercializadora.

- Decisión de que la gestión de ECASOL como coordinador de la cadena de suministro debe basarse en un amplio uso de la tercerización y no en el aumento de la infraestructura de almacenes y medios de transporte administrados por la Empresa. Se establece, como principal operador logístico a utilizar a

- Almacenes Universales S. A., fundamentalmente en los servicios de almacenamiento y transportación.

- Primeros análisis de capacidad en los procesos productivos de aceite permitiendo obtener las primeras aproximaciones a los problemas reales a enfrentar en la fábrica de Regla, en vistas a mejorar la disponibilidad y el servicio al cliente final.

- Segunda representación de la Cadena de Suministro del Aceite Comestible Comercializado en CUC, donde se presentan 27 niveles y se confecciona un único concepto de Cadena de Valor que guía en lo adelante el funcionamiento de la misma. Se definen como objetivos de mejoramiento de la Cadena de

- Valor: aumento de la disponibilidad del producto en mercado, incremento de la eficiencia y competitividad e incremento de las ventas.

- Queda fijado como objetivo fundamental de trabajo de la cadena de suministro el garantizar un nivel de disponibilidad del aceite, como genérico, en los puntos de venta de las cadenas comercializadoras del $90 \%$.

\section{II.2.2 SEGUNDA ETAPA (2008-2010)}

- Redefinición de la composición de la cadena de suministro del aceite comestible en CUC en función de los eslabones esenciales para la gestión e integración de la cadena, quedando conformada la misma por 7 eslabones y 19 actores principales.

- Redefinición del objeto social de la empresa ECASOL para facilitar su papel como coordinador y desarrollador de la cadena de suministro de aceite en CUC, constituyendo un precedente importante para el país.

- Estudio de los ciclos logísticos en la cadena de suministro y determinación de los subciclos con influencia en el incumplimiento de los niveles de servicio a los clientes.

- Profundización del estudio de las capacidades en el proceso de fabricación e inclusión de otros procesos de la cadena. Queda demostrada la validez de la metodología de cálculo y análisis de las capacidades en los sistemas logísticos, desarrollada por LOGESPRO. Este resultado constituye un referente para la aplicación de la metodología de cálculo de capacidades en cadenas de suministro.

- Obtención del Modelo de Aseguramiento del Proceso (MAP) de producción de aceite. Esto permite organizar y garantizar el nivel de abastecimiento requerido de materias primas, insumos y piezas de repuesto a la producción, lo que se traduce en una elevación de la continuidad de la producción y mejor aprovechamiento de las capacidades.

- Realización de la evaluación piloto de la aplicación del Modelo de Flujo logístico como herramienta de planificación coordinada de la cadena.

- Se alcanza el objetivo de mantener, con estabilidad, un nivel de disponibilidad del $90 \%$ en los puntos de ventas de las cadenas comercializadoras, lo cual se corrobora a partir de los resultados de los estudios de disponibilidad realizados por LOGESPRO y el MINCIN de forma independiente.

- Identificación de la necesidad de formalizar las relaciones de cooperación e integración entre los eslabones de la cadena de suministro, conformándose el esquema de organización de la misma.

- Queda evidenciada la necesidad de conformar y formalizar el proyecto de desarrollo integral de la cadena se suministro, pero con un alcance mayor al que se ha trabajado hasta esta etapa, se identifica la necesidad y posibilidad de incorporar nuevos eslabones, hacia atrás y hacia delante, en la gestión de la cadena. Hacia atrás llegando hasta la producción agrícola de la soya como principal materia prima y hacia adelante con la incorporación de otros productos que añadan valor en el marco de la cadena, tales como grasas de freído profundo y mayonesa. Se comienza a visualizar la cadena agroalimentaria de aceite, grasas vegetales y sus derivados comercializados en CUC.

\section{II.2.3 TERCERA ETAPA (2010-2013)}

- Estudio de la factibilidad de incorporar a la cadena la comercialización de la mayonesa "Doña Delicias" en coordinación con el MINAL, bajo un esquema similar al que se tiene con la comercialización de aceite.

- Estudio de la factibilidad de la producción y comercialización de grasas de freído profundo con el objetivo de satisfacer necesidades no resueltas en el mercado y otras que deben surgir con el incremento del trabajo por cuenta propia, específicamente en el caso de la elaboración de alimentos.

- Estudio de la incorporación del tratamiento de los residuales a la cadena (logística reversa) y búsqueda de alternativas para agregar valor a la misma a partir de la comercialización y/o reprocesamiento de las grasas quemadas y residuales de la refinación de aceite.

- Se trabaja en el desarrollo del mecanismo de coordinación de la cadena, sustentado en garantizar el equilibrio entre las capacidades y el flujo logístico en los diferentes eslabones. Se fundamenta el mecanismo en los modelos de cálculo y análisis de las capacidades y de flujo, desarrollados por LOGESPRO.

\section{II.2. 4 ETAPA ACTUAL (2013-ACTUALIDAD)}

- A partir de las experiencias obtenidas en las etapas anteriores y la aprehensión paulatina de los conceptos de logística y cadenas de suministros en la empresa, en la actualidad se está manejando el desarrollo de una Cuarta Etapa, la cual está centrada 
en la innovación, siendo la base para el surgimiento de las Redes de Valor [10].

- La Cadena de Suministro de Aceite Comercializado en CUC, con ECASOL como ente coordinador de la misma, transita hoy por los inicios de esta cuarta etapa de desarrollo. Como parte de esta etapa la cadena se traza como premisas las siguientes:

- Incorporación de nuevos eslabones hacia atrás llegando hasta la producción agrícola de soya como principal materia prima del proceso de obtención de aceite. En tal sentido se pretende incorporar de forma activa a esta cadena a la Planta Procesadora de Soya (PDS), la cual está radicada en Santiago de Cuba y constituye la única de su tipo en el país.

- Integración de la Cadena de Suministro de Aceite Comestible en Cuba, considerando las dos vertientes en que se desarrolla la misma, la rama de comercialización en CUC y la de comercialización en CUP.

- Consolidación de ECASOL como operador logístico de la Cadena de Aceite Comestible en Cuba.

\section{3 ACCIONES A DESARROLLAR EN LA NUEVA ETAPA POR ECASOL PARA EL CUMPLIMIENTO DE LAS PREMISAS}

\section{II.3.1 INTEGRACIÓN CON PDS}

Para el logro de la primera premisa ECASOL en su papel de ente coordinador se encuentra en la actualidad dando sus primeros pasos hacia la integración con la Planta Procesadora de Soya (PDS). PDS es una entidad que resulta en nuestro territorio, proveedor exclusivo de aceite crudo para la industria. Para el desarrollo de su proceso productivo, actualmente PDS gestiona el aprovisionamiento de materias primas, materiales e insumos del proceso que desarrolla, así como las piezas de repuesto de los equipos con que cuenta. Sus niveles actuales de producción de aceite crudo no cumplen las necesidades de refinación de aceite de la Refinadora de Aceite de Santiago de Cuba (ERASOL). Ante tal situación ECASOL pretende incorporar dicha entidad a su gestión con el objetivo de garantizar la estabilidad y continuidad del proceso de producción de aceite crudo.

\section{II.3.2 INTEGRACIÓN DE LA CADENA DE ACEITE NACIONAL}

Como se mencionaba al inicio del caso, los principales estudios relacionados a la comercialización de aceite en el país han estado encaminados fundamentalmente a la rama de comercialización en CUC. Hoy es un hecho la necesidad de lograr una integración más allá de este ámbito y es por tanto que se está comenzando a visualizar la integración de ambas vertientes en una única cadena de suministro a nivel nacional. Como parte de esta estrategia se está haciendo un levantamiento de manera conjunta entre ECASOL, el Grupo Empresarial de la

Industria Alimentaria (GEIA) y LOGESPRO de la Cadena de Suministro a nivel nacional integrando las ramas en CUC y CUP. El primer paso para el logro de esta premisa lo constituye la concepción de la Cadena de

Suministro de Aceite Comestible en el país, que cuenta hoy con un total de 15 eslabones verticales.

\section{II.3.3 ECASOL COMO OPERADOR LOGÍSTICO DE LA CADENA DE ACEITE}

Como base del desarrollo de ECASOL como operador logístico de la Cadena de Suministro Aceite a nivel nacional, la entidad se encuentra inmersa en la reestructuración de su organización. El cambio fundamental que se está llevando a cabo en la estructura consiste en la creación de una Dirección Logística que le permite concentrar de manera más efectiva la coordinación de todos los procesos relativos a la comercialización de aceite. Esta dirección es la antesala para que ECASOL comience a desempeñarse como operador logístico de la cadena.

\section{DESPLIEGUE DE LAS INVESTIGACIONES}

A lo largo de la labor desplegada entre ECASOL y LOGESPRO con el objetivo de elevar el nivel de servicio que la Cadena de Suministro de Aceite Comestible oferta a sus clientes, ha sido necesaria la incorporación paulatina de diferentes líneas de investigación, entre las cuales se encuentran:

- $\quad$ Estudios de disponibilidad en los puntos de venta.

- Análisis de capacidad en distintos eslabones de la cadena, fundamentalmente en los almacenes de fábrica y en las líneas de envase.

- Evaluación de diferentes alternativas de envases para la comercialización del aceite.

- Diseño del proceso de aprovisionamiento de materias primas y materiales a las industrias aceiteras.

- Evaluación de la reutilización de desechos en la cadena.

- Aplicación del Modelo General de Organización (MGO) en la empresa ECASOL.

- Implementación del Modelo de Diseño de los Nodos de Integración en la Cadena de Suministro

- (MDNICS) en puntos críticos, tales como el proceso de despacho de aceite en fábrica.

- Estudio del proceso de contratación entre los actores de la cadena.

- $\quad$ Ejecución del Modelo de Gestión Colaborativa del Flujo Logístico (MGCFL) en ECASOL.

En la figura 5 se puede observar un resumen de estas líneas por años. 


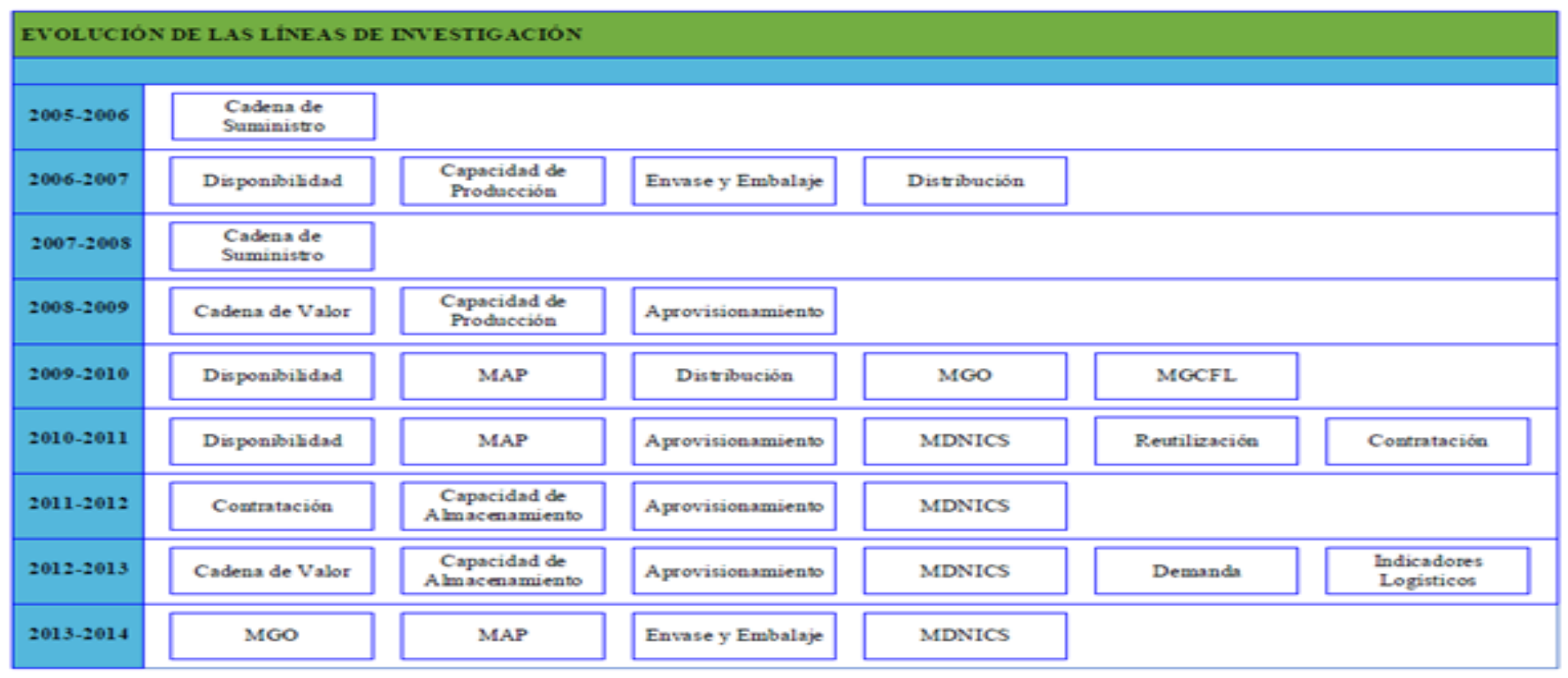

Figura 5: Evolución de las líneas de investigación desarrollas por ECASOL y LOGESPRO de manera conjunta.

Fuente: Los autores, (2018).

\section{SÍNTESIS DEL PROCESO EVOLUTIVO DE LA CADENA DE ACEITE COMESTIBLE EN EL PAÍS}

En la tabla que se muestra seguidamente aparece de manera resumida la evolución de la cadena de suministro de aceite comestible a lo largo del despliegue de las cuatro etapas de desarrollo que se han referido a lo largo del documento.

Tabla 1: Síntesis del desarrollo evolutivo de la cadena de aceite comestible en el país.

\section{Criterios}

Representación de la cadena de suministro

\section{Alcance de la gestión en la cadena}

\section{Enfoque en la CS}

Concepto de
disponibilidad

Meta de disponibilidad

\footnotetext{
Disponibilidad alcanzada por genérico
}

\section{Niveles de} integración

Etapa 1 (2005-2008)

Etapa 2 (2008-2010)

niveles verticales

y segunda con 27

niveles verticales

Rama de comercialización en CUC

No se tiene enfoque de CS, enfoque al interior de la empresa

\section{Disponibilidad} como genérico

Alcanzar un nivel de disponibilidad del aceite como genérico del $90 \%$

$81,60 \%$

A nivel de cadena es de 2.83, Nivel Bajo
Se mantiene la

19 eslabones

Representación de 7 niveles eslabones

Rama de comercialización en CUC

Enfoque de CS

Disponibilidad genérico y por surtidos

Alcanzar un nivel de disponibilidad del aceite como genérico del $90 \%$

$84.17 \%$

No se estudia

No se estudia

Rama de CUC

$>99,55 \%$ verticales y 19
Etapa 3 (2010-2013) representación de 7 niveles verticales y

Etapa 4 (2013-actualidad)

Se representa la cadena de aceite comestible en Cuba con un total de 15 niveles verticales comercialización en

Cadena de suministro de aceite nacional (Incluye rama de CUC y CUP)

Enfoque de CS con énfasis en los nodos de integración de la CS con ampliación del alcance de la CS

Disponibilidad genérico y por surtidos

Alcanzar y mantener un valor de $90 \%$ a nivel de surtido de disponibilidad del aceite como genérico del $90 \%$

$>99,55 \%$

Evaluación media de 2,65 puntos, Nivel Bajo 
Baez et al., ITEGAM-JETIA. Vol. 04, № 13, pp 127-134. March, 2018.

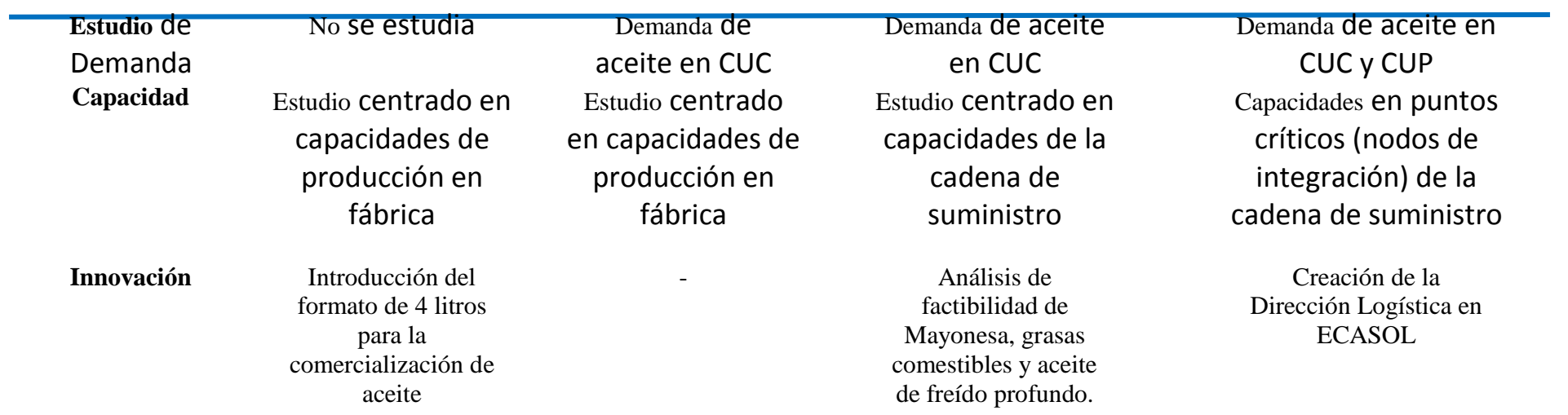

Fuente: Los autores, (2018).

Como se aprecia en la tabla 1 en la Etapa 4 el nivel de integración es inferior al de la Etapa 1, lo cual está dado por cambios de dirección que se producen en el año 2012, afectando el pilar liderazgo al cual se hizo alusión con anterioridad.

\section{CONCLUSIONES}

El análisis de este caso permite, a modo de resumen, relacionar las enseñanzas siguientes:

La aplicación paulatina del Modelo de Red de Valor en la Cadena de Suministro de Aceite Comestible en CUC ha propiciado un aumento considerable en la disponibilidad de aceite en un $73,15 \%$ y las ventas en 31402 MCUC.

El éxito del desarrollo de la GICS debe estar apoyado en un proceso de fomento de la preparación del personal, el desarrollo de la cultura empresarial y en el liderazgo de la alta dirección de la empresa en tal empeño.

La relación universidad-empresa permite la integración de elementos teórico-prácticos en la mejora de la gestión empresarial.

Las etapas de la GICS no son estáticas pudiendo darse retrocesos en los avances obtenidos, lo cual conduce a un proceso de replanteamiento de acciones de etapas anteriores.

\section{RECONOCIMIENTOS}

Los autores agradecen a los empresarios, operarios y estudiantes que han colaborado con la realización de la investigación, en especial al personal de la empresa ECASOL.

\section{REFERENCIAS}

[1] BAEZ, Y.P., Modelo de Diseño de Nodos de Integración en la Cadena de Suministro bajo las condiciones cubanas, in Departamento de Ingeniería Industrial. 2011, Instituto Superior Politécnico José Antonio Echeverría Habana.

[2] AGUILAR, P.R., Administración de la Cadena de Suministro (SCM). Lo que todos deberían saber. 2009.

[3] MANGAN, J., C. LALWANI, and T. BUTCHER, Global Logistics and Supply Chain Management, ed. J.W.a. Sons. 2008.

[4] ANAYA, J.J. and S.P. MARTÍN, Innovación y mejora de procesos logísticos: Análisis, diagnóstico e implementación de sistemas logísticos. 2da ed, ed. ESIC. 2007, Madrid.

[5] FLYNN, B.B., B. HUO, and X. ZHAO, The impact of supply chain integration on performance: $A$ contingency and configuration approach. Journal of Operation Managements, 2010. 28: p. 58-71.

[6] ACOSTA, M.I.G. and J.A.A. SUAREZ, La Logística Moderna en la Empresa, ed. F. Varela. 2010, Habana.

[7] IVANOV, D. and B. SOKOLOV, Adaptive Supply Chain Management. 2010, London: Springer.

[8] VITASEK, K., Supply Chain Management Terms and Glossary. 2009, Bellevue, Washington: Supply Chain Visions.

[9] HARRISON, A. and R.V. HOEK, Logistics Management and Strategy. Competing through the supply chain, ed. P. Hall. 2008, London.

[10] SUAREZ, J.A.A., Modelos y estrategias de desarrollo de la Logística y las Redes de Valor en el entorno de Cuba y Latinoamérica. 2008, Instituto Superior Politécnico José Antonio Echeverría: Ciudad de la Habana.

[11] CUBA, Lineamientos de la Política Económica y Social del Partido y la Revolución. 18 de abril 2011, VI Congreso del Partido Comunista de Cuba.

[12] BAEZ, Y.P. and A.J.A. URQUIAGA, Estudio de disponibilidad de aceite comestible en Cuba para el año 2010. Ingeniería Industrial, 2012. 33(1): p. 94-97.

[13] BAEZ, Y.P. CASO: ECASOL. Cadena de Suministro de aceite comestible y sus derivados en Cuba. 2013. Empresa Comercializadora de Aceites ECASOL.

[14] SUAREZ, J.A.A. and M.I.G. ACOSTA, ¿Mi empresa o la cadena de suministro? Nuevo dilema del directivo. Nueva Empresa, 2012. 8.

[15] MINISTROS, C.D., Reglamento para la Implantación y Consolidación del Sistema de Dirección y Gestión Empresarial Estatal in 41. 2007, Gaceta Oficial. 